This is a peer-reviewed, accepted author manuscript of the following article:

Joannidis, C. A., Haddrill, P. R., \& Laing, K. (2020). Determination of the most effective

enhancement process for latent fingermarks on Clydesdale Bank and Royal Bank of

Scotland £5 and £10 polymer banknotes. Forensic Science International, [110334].

https://doi.org/10.1016/j.forsciint.2020.110334

\title{
Determination of the Most Effective Enhancement Process for Latent Fingermarks on Clydesdale Bank and Royal Bank of Scotland £5 and £10 Polymer Banknotes
}

Carina Anna Joannidis (corresponding author) ${ }^{1}$

carinajoannidis@gmail.com (carina.joannidis@spa.pnn.police.uk)

Centre for Forensic Science

Department of Pure and Applied Chemistry

University of Strathclyde

204 George Street

Glasgow

G1 1XW

Penelope R. Haddrill

penny.haddrill@strath.ac.uk

Centre for Forensic Science

Department of Pure and Applied Chemistry

University of Strathclyde

204 George Street

Glasgow

G1 1XW

Kenny Laing

Kenny.Laing@spa.pnn.police.uk

SPA Forensic Services

Scottish Crime Campus

\footnotetext{
${ }^{1}$ Present Address: SPA Forensic Services, Scottish Crime Campus, Craignethan Drive, Gartcosh, G69 8AE
} 


\section{Craignethan Drive}

Gartcosh

G69 8AE 


\section{Highlights}

- Clydesdale Bank and Royal Bank of Scotland $£ 5$ and $£ 10$ polymer banknotes examined

- PolyCyano UV followed by black magnetic powder provided most effective enhancement

- Black powder suspension was also effective on all note types

- IR light and blue Crime-lite improved visualisation of fingermarks 


\section{Abstract}

Fingermarks are commonly found at crime scenes and can be used to link an individual to an object and/or place. One common evidence type regularly encountered in the course of a criminal investigation is banknotes, and the recovery of fingermarks from these notes can give an indication of who has handled them. This study was carried out in order to determine the most effective sequential processing techniques for recovering latent fingermarks on the new $£ 5$ and $£ 10$ Clydesdale Bank and Royal Bank of Scotland polymer banknotes. No previous studies have been published on the recovery of latent fingermarks from these types of polymer notes; therefore, this work provides valuable insight into the challenges associated with these notes. Initial experimentation was done in order to determine the best light source to be used in combination with each sequential process tested. From this, infrared $(730-800 \mathrm{~nm})$ and ultraviolet (350- $380 \mathrm{~nm}$ ) light were chosen for use in the main study. Black iron oxide powder suspension and black magnetic powder were two of the enhancement treatments tested, both of which are recommended 'Category A' processes in the Fingermark Visualisation Manual produced by the Home Office. Superglue fuming - using PolyCycano UV - which is a Category $C$ process was also used, as well as the recently developed infrared fluorescent powder, fpNATURAL ${ }^{2}$. Three fingermark donors were selected for this study-one good, one medium and one poor-and each donor deposited a 10-mark depletion series onto both sides of each type of note. Superglue fuming (using PolyCyano UV) followed by black magnetic powder was found to be the most effective sequential process for enhancement of fingermarks on all note types tested. Infrared (730-800 nm) light with an $815 \mathrm{~nm}$ filter was the most effective light source for enhancing ridge detail for this enhancement sequence. This process is now being implemented for use with these note types in Scotland.

\section{Keywords}

Polymer banknotes, PolyCyano UV, Infrared, Black magnetic powder, Powder suspension, fPNATURAL $\circledast 2$ 


\section{Introduction}

Since the late $19^{\text {th }}$ century, fingerprints have been a valuable source of identification in forensic investigations [1]. They are valuable because they can be individualised, remain unchanged throughout life and can only be disfigured as a result of deep scarring [2, 3, 4]. In any criminal investigation a suspect can be linked to an object and/or place by the presence of their fingermarks [2]. A common example of this would be fingermarks recovered from banknotes used in criminal activity. The recovery of fingermarks from banknotes has changed considerably over the last 30 years, as a result of the introduction of polymer notes.

Australia introduced the first polymer banknotes in 1988, and they have been released in many countries during the three decades following this. Polymer banknotes are now in circulation in more than 30 countries [5] and the composition and processes used to produce polymer banknotes vary between them $[6,7,8,9]$. In the UK, Clydesdale Bank (CB), the Royal Bank of Scotland (RBS) and the Bank of Scotland all released a $£ 5$ polymer banknote in Scotland in 2016, followed by a $£ 10$ polymer banknote in 2017. The Bank of England (BoE) also released their $£ 5$ and $£ 10$ polymer banknotes in England within the same timeframe $[6,10]$. This change in material from the old cotton notes was implemented in order to increase counterfeit resilience and durability $[6,11]$.

The detection and enhancement of latent fingermarks is an important tool in the identification of any individuals involved in the handling of evidence [6]. Understanding the best techniques for the recovery of latent prints on polymer banknotes is therefore crucial in criminal investigations [6]. Unfortunately, conventional mark enhancement techniques that are recommended for use on the old cotton banknotes are not effective on new polymer notes. This is due to the non-porous nature of these polymer notes in comparison to cotton paper notes [6]. Previous research has also reported differences in fingermark recovery between the opaque areas of the notes and the transparent windows $[6,7,12]$. Studies on both Australian and Canadian banknotes reported cyanoacrylate fuming to be the most successful technique for developing marks on the transparent areas of the notes [7, 12].

The Scottish Police Authority (SPA) Forensic Services can receive hundreds of thousands of pounds' worth of banknotes during the investigation of a single case. The majority of these are Clydesdale banknotes, as they are the most common notes in circulation in Scotland. Due to the large number of notes received as evidence, SPA's forensic scientists require an efficient and effective method of retrieving latent fingermarks from these notes. The newly released polymer banknotes are made of bi-axially orientated polypropene (BOPP) $[6,11]$. 
This material makes the banknotes semi- or non-porous [2]. Furthermore, these polymer banknotes are particularly challenging substrates to work with due to the large amount of background pattern interference and their lack of surface uniformity [13]. Whilst the Bank of England notes appear to have a very smooth polymer coating covering the whole note, Scottish banknotes appear to have more textured areas and a less smooth finish.

Currently, there are a number of chemical (e.g., superglue fuming), physical (e.g., powder) and optical (e.g., ultraviolet) processes available for fingermark enhancement, and the quality of the mark recovered may be improved by using a combination of processes [14]. Enhancement techniques are selected depending on three individual factors: the surface on which the fingermark is deposited, the composition of the fingermark residue, and the environmental conditions that the mark has been exposed to [15, 16]. The Fingermark Visualisation Manual (FVM), developed by the Home Office, contains recommended techniques depending on the substrate type that the mark is found on [17]. Cyanoacrylate fuming and powder suspension are amongst the most common techniques used within the laboratory for the enhancement of latent prints on non-porous surfaces [2, 18].

Although there are many factors to consider when selecting an appropriate fingermark enhancement technique, it is recommended to use a sequence of processes [17, 19]. Regardless of the conditions a mark has been exposed to, using a sequential process will maximise recovery, as each process targets a different constituent within the fingermark. The FVM contains guidelines for selecting an appropriate sequence of processes, where the least destructive processes are used first (Figure 1). 
Optical processes

(non-destructive)

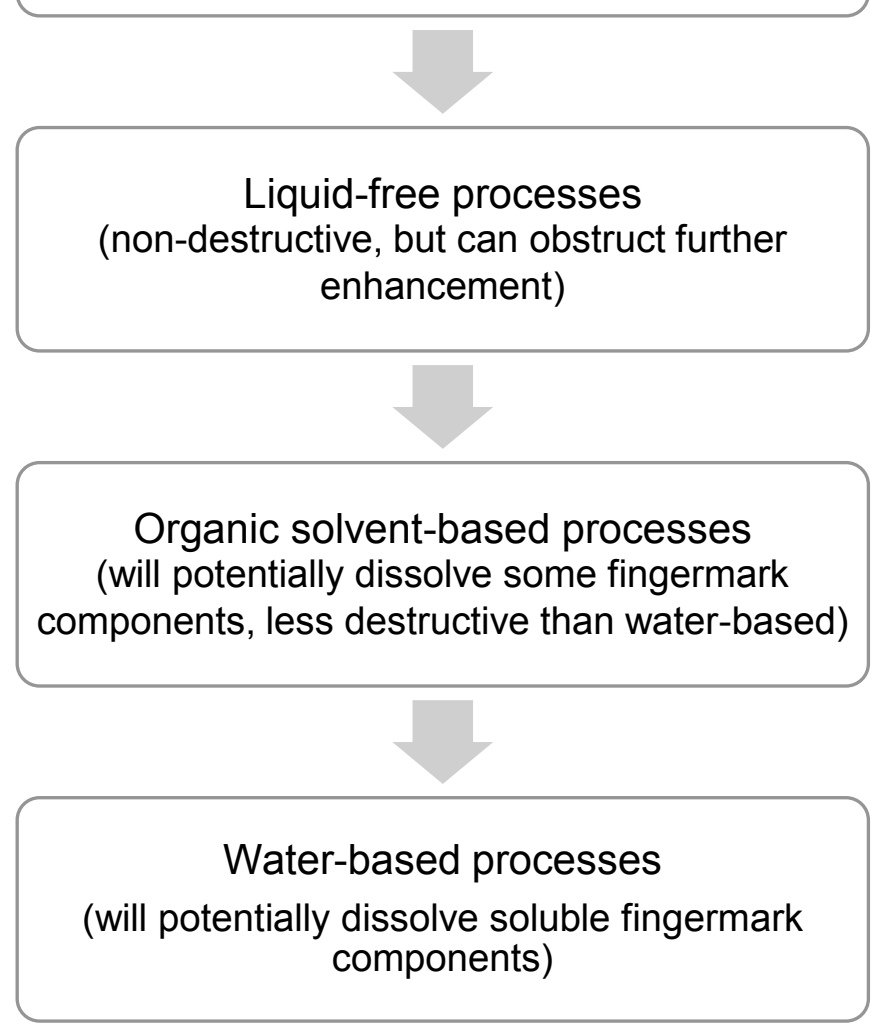

Figure 1. The recommended order of fingermark enhancement processes for the most effective results. Adapted from the Fingermark Visualisation Manual [17].

In 2016, the Home Office and collaborators carried out a study testing fingermark visualisation techniques on uncirculated BoE $£ 5$ polymer banknotes [6], and this was later expanded in 2017 to look at BoE $£ 10$ polymer banknotes [13]. The study used three fingermark enhancement sequences, which were applied to 64 fingermarks deposited onto eight polymer banknotes. The fingermarks were separated into two age ranges: 2-3 days and 12-13 days $[6,13]$. The study concluded that the two most effective sequences for visualising fingermarks were fpNATURAL®2 followed by fpNATURAL®2 powder suspension, or magnetic powder followed by iron oxide powder suspension. The results of this study have been incorporated into the Home Office guidelines for fingermark visualisation [20]. These guidelines advise the best sequence of processes to visualise fingermarks on BoE polymer banknotes (see Figure 2). However, no previous research has been carried out to investigate which enhancement processes are most effective on CB and RBS polymer notes. The SPA Forensic Services must follow the guidelines published by the Home Office or carry out their own internal validation. 
The FVM recommendation for fingermark enhancement on polymeric currency includes an initial visual search followed by a fluorescence examination. Thereafter either superglue fuming > vacuum metal deposition $>$ superglue fluorescent dye staining $>$ basic violet 3 , or black powder suspension > basic violet 3 can be used (although the effectiveness of powder suspension and basic violet 3 is unknown) [17]. The Home Office guidelines along with the FVM have therefore been used on Scottish polymer notes since their release in 2016, but both have been unsuccessful in fingermark recovery. Although the uncontrolled nature of fingermarks deposited in casework conditions leads to high levels of variability in the effectiveness of fingermark recoverey, further research examining the variation in recovery and the effectiveness and sensitivity of these processes is required [13].

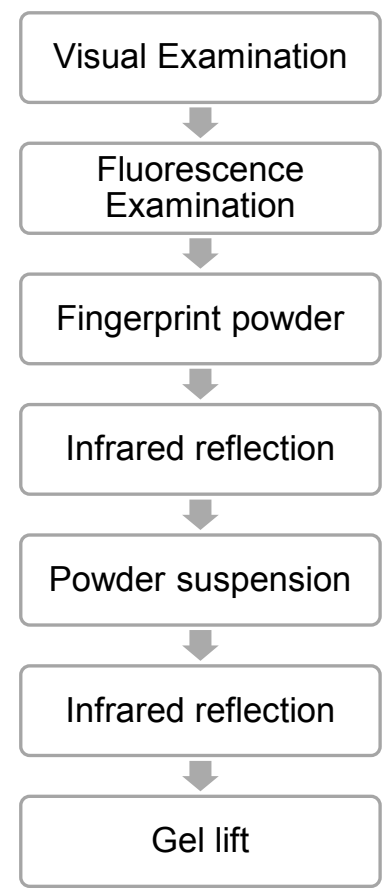

Figure 2. Sequential processing recommended by the Home Office for use on Bank of England polymer notes [20].

The FVM also includes recommendations for light sources that can be used to aid in visualisation of fingermarks. Infrared reflection is an optical process that is useful for enhancing fingermarks present on patterned or coloured substrates, such as banknotes. This optical process involves simultaneously using an IR radiation light source to illuminate the fingermark and an IR sensitive imaging system to view the effects. This technique, which is non-destructive and has no detrimental impact on the fingermark, is useful for visualising marks on complex substrates as it can remove background interference [19, 21]. Downham 
et al. found this technique to be useful in reducing the background patterns on the BoE $£ 5$ notes via reflection and transmission of incident near IR (NIR) radiation [6]. Ultraviolet imaging is similar to IR, as it creates a contrast between the fingermark and substrate. Similar to the method above, the illumination of the mark with short wavelength radiation causes electrons within fingermark constituents to enter an excited state. To return to their initial ground state, the electrons must lose energy, which is emitted as a photon of light (i.e., fluorescence).

The aim of this study was to determine the most efficient mark enhancement technique(s) for the development of latent fingermarks on Scottish polymer banknotes. The notes used were obtained directly from CB and RBS. Each bank provided new uncirculated banknotes, which were a mixture of $£ 5$ and $£ 10$ notes. Cyanoacrylate fuming (using PolyCyano UV), powder suspension, magnetic powder and IR powder (fpNATURAL $® 2$ ), which are believed to work well with the specific surface features of polymer banknotes, were used in this study. These processes were used in conjunction with different light sources and light filters to determine the best techniques for use with these banknotes.

\section{Materials and methods}

\section{1 Preliminary study}

Preliminary testing was conducted to determine the best light source for use in combination with each enhancement technique and this was incorporated into the processing sequences. A range of light sources - both single and multiple sources (see below) - were tested to determine which, if any, could filter out the background patterns. All light sources were used to illuminate the banknotes and photographs were taken. All photographs were taken using a Nikon D5 camera, with an AF-S VR Micro-Nikkor $105 \mathrm{~mm}$ lens mounted to a copystand. Infrared (730-800 nm), UV (350 - $380 \mathrm{~nm})$, white light and various coloured lights (410 nm, $445 \mathrm{~nm}, 475 \mathrm{~nm}, 520 \mathrm{~nm}, 590 \mathrm{~nm}$ and $640 \mathrm{~nm}$ ) were tested in this preliminary study, along

with a number of colour filtration filters $(693 \mathrm{~nm}, 751 \mathrm{~nm}, 815 \mathrm{~nm}, 859 \mathrm{~nm}$; Foster + Freeman Anti-Glare Schott) that were fitted onto the camera lens. All settings were altered via the Foster + Freeman DCS $® 5$ imaging system on a computer, to which the camera was connected. Instead of using handheld Crime-lites for the different wavelengths a multi-wavelength photographic ring light (Crime-lite ${ }^{\circledR} 8 \times 4 \mathrm{MK} 2$; Foster + Freeman) was fitted onto the copystand. All images captured under IR light required a long shutter speed (15-20 seconds) for an image to be visible. Figure 3 shows the effects of different light sources on the CB $£ 10$ polymer note. 
a)

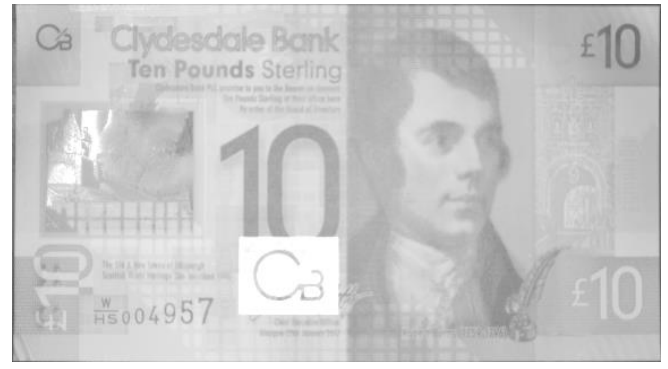

c)

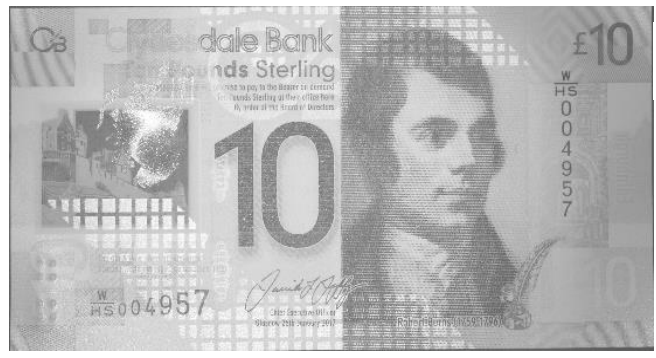

e)

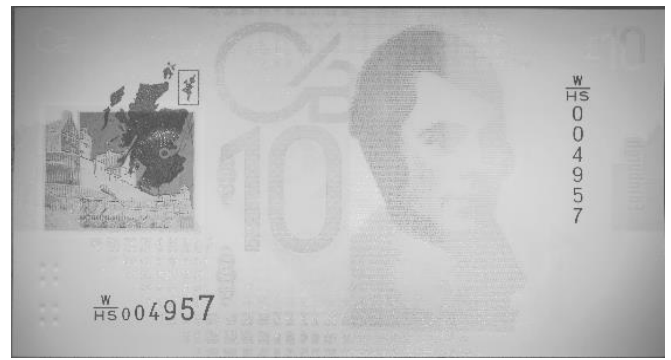

b)

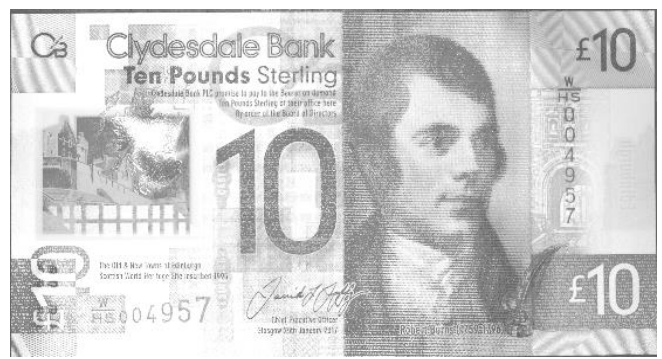

d)

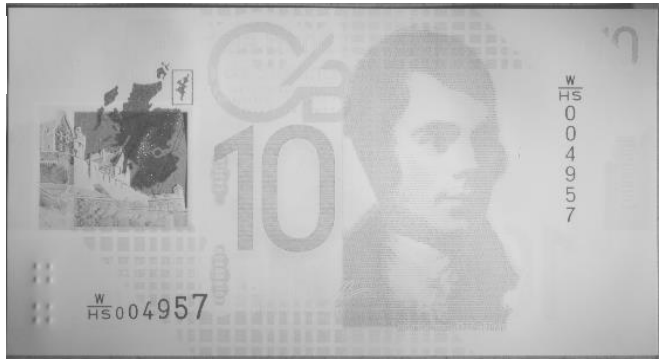

f)

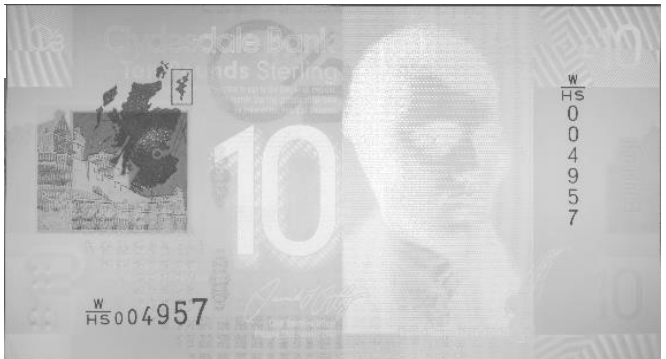

Figure 3. Clydesdale Bank $£ 10$ note photographed under different light conditions: a) UV light $(350-380 \mathrm{~nm})$ b) Blue (445 nm), c) Yellow (590 nm), d) IR (730-800 nm), e) IR $(730-800 \mathrm{~nm})+815 \mathrm{~nm}$ filter and f) IR $(730-800 \mathrm{~nm})+815 \mathrm{~nm}$ filter + Blue $(445 \mathrm{~nm})$ Crime-lite (30\%).

A number of enhancement techniques were considered during the preliminary study. Due to the semi porous nature of the polymer banknotes, treatments used on porous items, such as ninhydrin and 1,8-diazafluoren-9-one (DFO) were not considered. Category C process PolyCyano UV was chosen over standard superglue fuming followed by a dye. Both processes were tested on banknotes during the preliminary study and PolyCyano UV was found to give better results. As the polymer notes are semi-porous, the tested dye (Basic Yellow 40; BY40) caused staining to areas of the notes that contained heavy black ink printing. Although PolyCyano UV is named a Category C process in the FVM, internal studies [22, 23] have been carried out within SPA that show it is equally effective and more efficient than standard superglue fuming followed by BY40. Furthermore, PolyCyano UV is commonly used in casework due to its superior performance and because it is a one-step process. The UV 
fluorescent security features within the banknotes were not significant enough to obliterate any fingermarks; therefore, the use of UV light (350-380 $\mathrm{nm}$ ) was not detrimental.

The treatments were selected based on resources available to all criminal justice partners in the United Kingdom (UK). The purpose of this study was to find the best enhancement treatment that could be used operationally; therefore, all treatments and technologies used had to be common to all police forces in the UK without the need to outsource for special equipment. Vacuum metal deposition (VMD) has been successful in recovering fingermarks on Canadian and Australian polymer banknotes [7, 12]. However, not all forces own this type of equipment due to its size and cost. Furthermore, the use of VMD operationally on cases involving large quantities of banknotes would be time consuming and inefficient. During the preliminary study, black powder suspension was tested against white powder suspension, however, black powder suspension gave better results especially when viewed under IR light. Similarly, fpNatural $\AA_{1}$ was tested against fpNatural $\circledast 2$, however, fpNatural@1 did not enhance any ridge detail. Both white powder suspension and fpNatural $(1$ were therefore not selected for use in the main study.

Table 1 shows each of the selected processing sequences used and the corresponding light source selected. The most effective light source for each process was used throughout the remainder of this study. No previous studies have been published on the effect of using a mixture of light sources on the enhancement of fingermarks. However, this study found that, for example, the effect of using an IR light $(730-800 \mathrm{~nm})$ with an $815 \mathrm{~nm}$ light filter and blue Crime-lite (445 nm), set at 30\% (adjusted using theDCS®5 imaging software), gave the most effective results for the processing sequence of IR fluorescent power and black powder suspension. The purpose of testing multiple light sources was to determine what conditions would make fingermark visualisation the easiest, and initial testing demonstrated that different light sources aided in the reduction of different features of the banknotes. Although using a combination of light sources is not common practice, this method is regularly used by SPA staff in order to generate the best possible fingermark visualisation results. 
Table 1. Enhancement processes used in this study and their corresponding light sources

\begin{tabular}{|c|c|c|c|c|}
\hline Sequence & $\begin{array}{c}\text { Chemical name } \\
\text { (abbreviation) }\end{array}$ & Manufacturer & Light Source & Light filter \\
\hline Superglue fuming & $\begin{array}{c}\text { PolyCyano UV } \\
\text { (Poly) }\end{array}$ & $\begin{array}{l}\text { Foster + } \\
\text { Freeman }\end{array}$ & $\begin{array}{l}\text { Ultraviolet (UV } \\
350-380 \mathrm{~nm} \text { ) }\end{array}$ & - \\
\hline $\begin{array}{c}\text { Infrared } \\
\text { fluorescent } \\
\text { powder }\end{array}$ & $\begin{array}{c}\text { fpNATURAL®2 } \\
(\mathrm{N} 2)\end{array}$ & $\begin{array}{l}\text { Foster + } \\
\text { Freeman }\end{array}$ & $\begin{array}{c}\text { Infrared }(730-800 \\
n m)\end{array}$ & $\begin{array}{c}815 \mathrm{~nm} \text { (long- } \\
\text { pass) }\end{array}$ \\
\hline $\begin{array}{c}\text { Powder } \\
\text { suspension }\end{array}$ & $\begin{array}{c}\text { Black powder } \\
\text { suspension (PS) }\end{array}$ & WhitChem & $\begin{array}{l}\text { Infrared (730- } \\
\quad 800 \mathrm{~nm})\end{array}$ & - \\
\hline \multirow{2}{*}{$\begin{array}{c}\text { Infrared powder + } \\
\text { Powder } \\
\text { suspension }\end{array}$} & fpNATURAL®2 & $\begin{array}{l}\text { Foster + } \\
\text { Freeman }\end{array}$ & \multirow{2}{*}{$\begin{array}{l}\text { Infrared }(730-800 \\
\mathrm{nm})\end{array}$} & $\begin{array}{l}815 \mathrm{~nm} \text { (long- } \\
\text { pass) + blue }\end{array}$ \\
\hline & $\begin{array}{l}\text { Black powder } \\
\text { suspension }\end{array}$ & WhitChem & & $\begin{array}{c}\text { Crime-lite (445 } \\
\mathrm{nm}, 30 \%)\end{array}$ \\
\hline \multirow{2}{*}{$\begin{array}{l}\text { Superglue fuming } \\
+ \text { Black magnetic } \\
\text { powder }\end{array}$} & PolyCyano UV & $\begin{array}{l}\text { Foster + } \\
\text { Freeman }\end{array}$ & \multirow{2}{*}{$\begin{array}{c}\text { Infrared }(730-800 \\
\mathrm{nm})\end{array}$} & \multirow[b]{2}{*}{$\begin{array}{c}815 \mathrm{~nm} \text { (long- } \\
\text { pass) }\end{array}$} \\
\hline & $\begin{array}{c}\text { SupraNano } \\
\text { black magnetic } \\
\text { powder (BP) }\end{array}$ & Scenesafe & & \\
\hline
\end{tabular}

Preliminary testing was also carried out on a variety of different substrate types, using five donors, who ranged in age from 20-50 years, and were a mixture of males (three donors) and females (two donors). This was done in order to find three donors for use in the main study who were known to be poor, medium and good donors, who gave consistent results across all five processing techniques or sequences and on all note types.

Fingermarks were deposited by the five donors ( $A$ to $E$ ) onto the non-adhesive side of brown sticky tape, a white polyvinyl chloride (PVC) board, black PVC board and an aluminium board (all purchased from $B \& Q$ ), and the different enhancement techniques applied. All donors deposited a 10-mark depletion series of natural fingermarks (i.e., donors did not rub their fingers on their forehead to build up sebaceous material), per substrate, at the same time of day, and 30 minutes after washing their hands, to ensure a good quantity of cellular material had built up for deposition in their marks, in line with guidance set out by the International 
Fingerprint Research Group [24]. The quality of each fingermark was evaluated by the clarity of ridge marks present as well as the percentage of the fingermark that was visible. On the basis of this, three donors were selected for the main study as poor (donor A), medium (donor E) and good (donor C). Split fingermark testing was used in the preliminary study on the brown sticky tape in order to test different enhancement techniques on the same fingermark. The banknotes used within this study were to be returned to the banks after use and permission was not given to destroy the banknotes, therefore split fingermark testing was not used in the main study for comparison purposes.

\subsection{Main study}

\subsubsection{Substrates}

CB and RBS $£ 5$ and $£ 10$ polymer banknotes were tested in this study. Each bank provided 144 uncirculated new notes. These notes were used during the study to control the area and time since deposition of each fingermark. All materials used were handled using disposable nitrile gloves (Ansell, Melbourne, Australia) to prevent contamination.

\subsubsection{Depletion series}

A depletion series was used to determine the sensitivity of each method [24, 25]. A 10-box grid (Figure 4) was drawn on both sides of each banknote and each side of every note was categorised as side 1 or side 2 for scoring purposes (Figure 5). Side 1 and side 2 were kept consistent for every note to avoid the addition of another variable. Fingermarks were deposited progressively using the same finger on the same substrate. The further down the depletion that development is visible, the more sensitive the process is expected to be. 


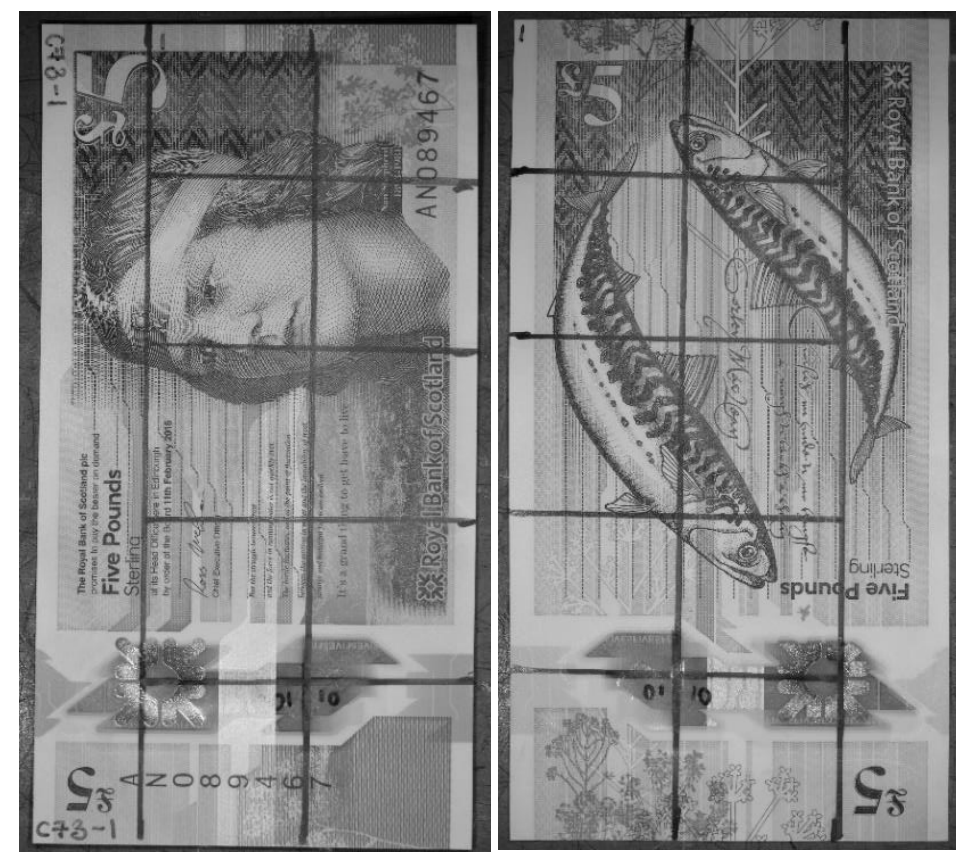

Figure 4. 10-box grid on both sides of a £5 RBS note. 
Side 1.
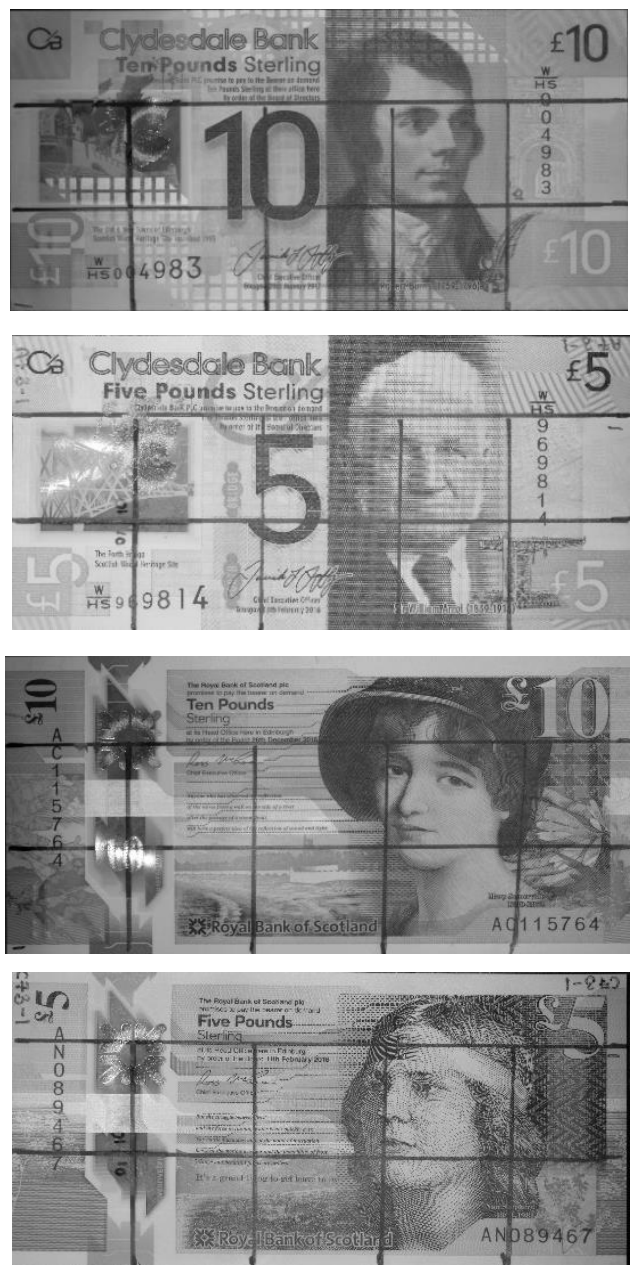

Side 2 .
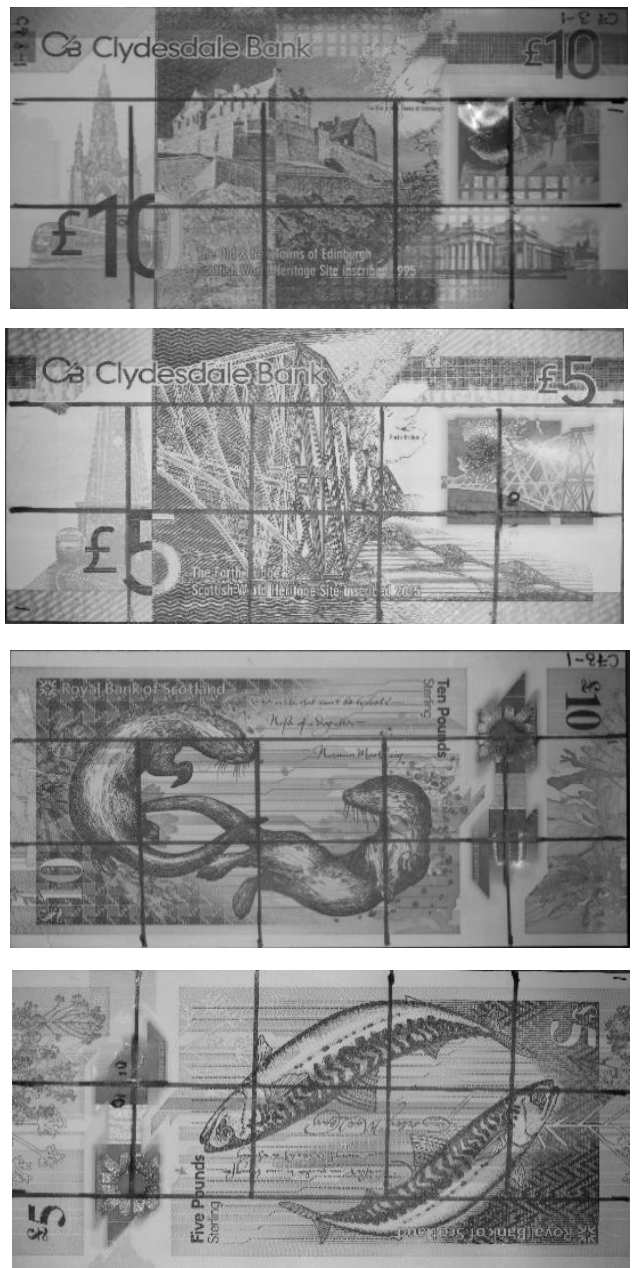

Figure 5. Indicates which side of each type of note was used as side 1 and side 2 for scoring purposes.

\subsubsection{Sample deposition}

There were 72 uncirculated notes of each type ( $£ 5$ CB, $£ 10$ CB, £5 RBS and £10 RBS) used in this study. The notes were separated equally into four different time periods: 7, 14, 21 and 28 days, to determine the effectiveness of each process on aged fingermarks [24]. On the first day (day 0), each donor deposited a depletion series onto both sides of 48 banknotes (12 of each type). A piece of tissue paper was placed onto the bench beneath the notes to prevent smudging. The notes for each donor were then divided equally ( 4 of each type) between each of the three processing sequences (Figure 6), before scoring. The entire experiment was repeated one day later, so that each sample in the study was duplicated, i.e., each donor deposited a depletion series onto both sides of a further 48 banknotes, which were split into the same categories as described above. 


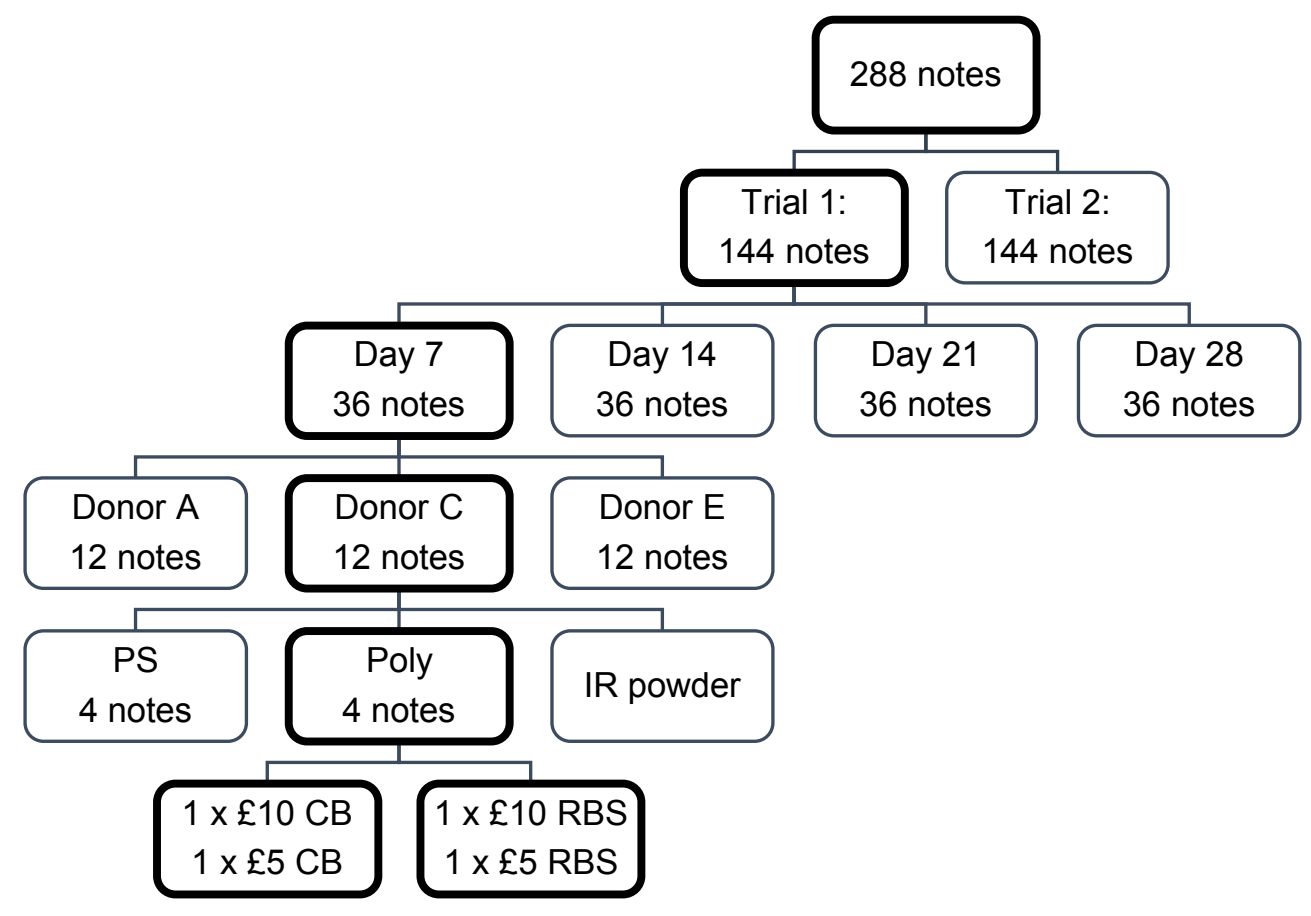

Figure 6. Outline of experimental design

\subsection{Enhancement sequences}

\subsubsection{PolyCyano UV - superglue fuming}

Cyanoacrylate fuming was carried out using PolyCyano UV powder (Foster + Freeman). Two level scoops of this powder (using the scoop provided with this product) were placed into an aluminium foil dish, which was then placed into a Foster + Freeman (Evesham, UK) MVC3000 fuming chamber, and distilled water added to the water dish located within the door of the chamber. The polymer notes being tested were suspended in the chamber using metal clips (Q-connect, Gent, Belgium), ensuring there was a gap between each note. The temperature was set to $230^{\circ} \mathrm{C}$, as this is the optimum temperature for fuming PolyCyano UV [26]. The fuming cycle was set to run for 20 minutes; however, this can be adjusted depending on the number of items being processed and the amount of development observed. Overall, the fuming process took approximately 1 hour to complete, including the following steps:

1. $15-$ to 20 -minute humidity cycle to reach optimum humidity $(80 \%)$.

2. 16- to 20- minute fuming cycle.

3. 15- to 20- minute purge cycle to decrease the humidity and temperature as well as removing toxic chemicals from the fuming chamber before opening. 
The cyanoacrylate vapours formed during the fuming cycle adhere to any fingermarks present on the notes, depositing a white coating. A strip of acetate was also hung in the chamber with known fingermarks placed onto it, as a positive control to ensure the process was working correctly. After the process was complete, the samples were removed from the chamber.

The notes were then examined and photographed in a dark room using a UV light (350 -380 $\mathrm{nm}$, Foster + Freeman), and the scores of all visible fingermarks were determined and recorded. Black magnetic powder (Scenesafe, Essex, UK) was applied to the marks several hours later, using a magnetic applicator, to determine whether this produced higher quality marks and/or helped enhance marks that were not previously visible. The notes were then photographed again, and the scores of all visible fingermarks were determined and recorded. For this second examination, an IR light (Crime-lite 730-800 nm, Foster + Freeman) was used, as this was found to give the best results with this combination of processes. Figure 7 shows the processing sequence used.

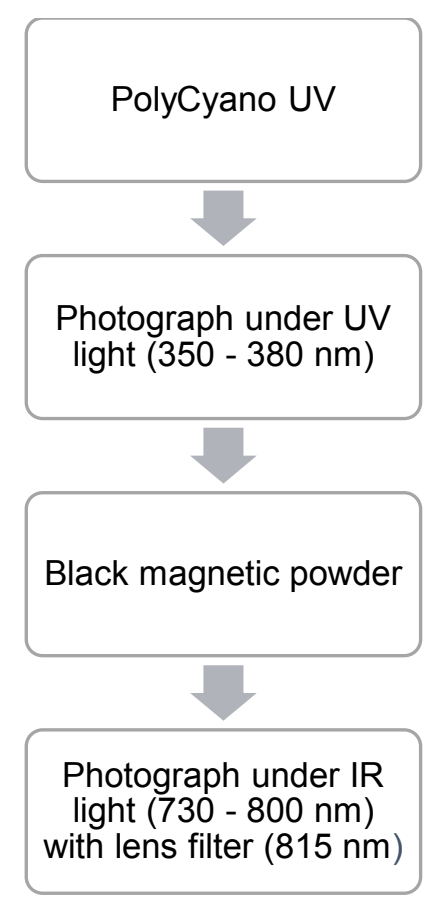

Figure 7. Flowchart for PolyCyano UV and PolyCyano UV + black magnetic powder 


\subsubsection{Powder suspension}

Powder suspension is believed to interact with the fatty constituents of fingermarks, resulting in dark or light fingermarks depending on which type of powder suspension is used (iron oxideand carbon-based or titanium dioxide-based, respectively) [17].The black powder suspension powder (50 g magnetic iron (II) Oxide, WhitChem, grade: precipitated (synthetic), magnetic, particle size: $200 \mathrm{~nm}-1 \mu \mathrm{m}$ ) was mixed with $500 \mathrm{~mL}$ ethylene glycol (Fisher Scientific, grade: $>99 \%$ ), $150 \mathrm{~mL}$ Triton X-100 (Fisher Scientific, grade: laboratory) and $1350 \mathrm{~mL}$ distilled water for 10 minutes (according to SPA's internal validated procedures). The suspension was applied to the banknotes over a sink using a soft brush. The solution was left on the banknotes for approximately 20-30 seconds and then washed off with tap water using a plastic hose. The notes were then hung up using metal clips and left to air dry for 2-3 hours at room temperature. Once the notes were dry, they were examined and photographed using IR light (Crime-lite $730-800 \mathrm{~nm}$, Foster + Freeman) and the scores of all visible fingermarks were determined and recorded. Figure 8 shows the processing sequence used.

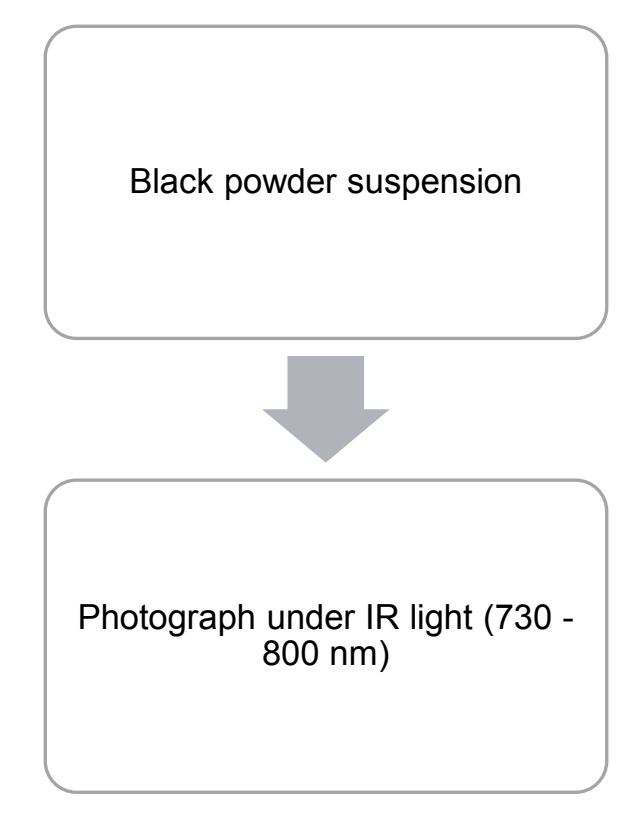

Figure 8. Flowchart of processing sequence used for powder suspension

\subsubsection{IR fluorescent powder (fpNATURAL®2)}

The fpNATURAL®2 powder (Foster + Freeman) was dusted onto the banknotes gently using a zephyr brush, and any excess powder was shaken or dusted off using a clean brush. The notes were then examined and photographed under IR light (Crime-lite IR 730-800 nm, Foster + Freeman) and the scores of all visible fingermarks were determined and recorded. 
Following this, powder suspension was applied to the notes (as described in section 2.3.2), to determine whether this produced higher quality marks and/or helped enhance those marks that were not previously visible. The notes were then photographed again, and the scores of all visible fingermarks were determined and recorded. Figure 9 shows the processing sequence used.

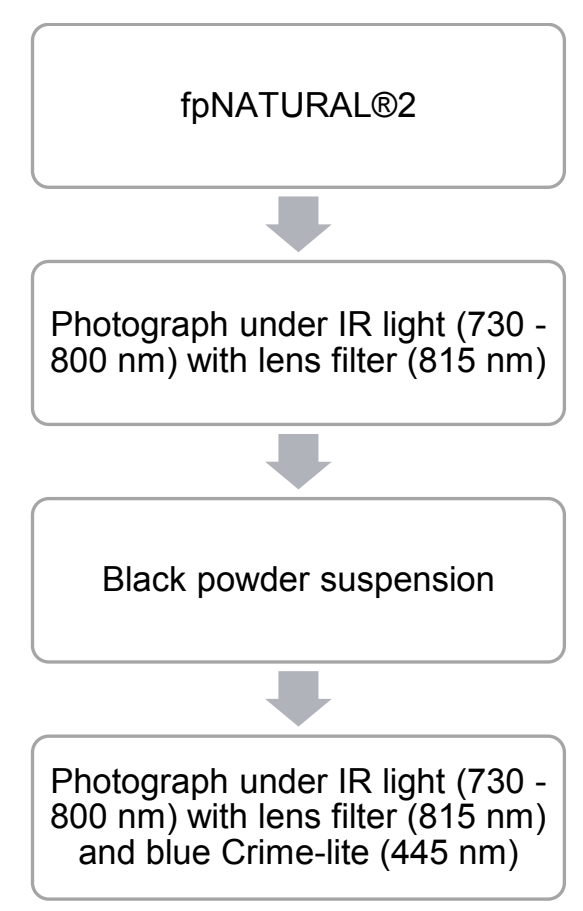

Figure 9. Flowchart of processing sequence used for fpNATURAL $B 2$ and fpNATURAL $B 2+$ powder suspension

\subsection{Scoring}

In order to statistically analyse the effectiveness of each enhancement technique, the quality of all fingermarks was assigned a numerical value [25]. Thirty-five years ago, the Home Office implemented a grading system that allows a numerical score to be given to a fingermark [25]. The grading corresponds to the proportion of features visible in each mark (Table 2). However, this method is subjective, therefore scoring of all marks was carried out by the same examiner under the same laboratory conditions. Due to the use of IR, scoring was performed from a digital capture of each depletion series, viewed on a computer screen with no digital enhancement for consistency [25]. 
Each of the ten marks in each depletion series was given a score between 0 and 4 . The scores across each depletion series were then added together to give total score out of 40 (10 marks with a maximum score of 4 each). A second score, out of 10 , was also given to each depletion series. This score indicated how many of the ten fingermarks gave a score of 1 or more, i.e., the number of samples that contained any evidence of a fingermark. The score given out of 40 could be considered to be subjective, whereas the score out of 10 is not. This second score was included for comparison to the score out of 40 , in order to determine the impact of any subjectivity on the results.

Table 2. The description given to each score between 0 and 4, as described by the Home Office [25].

\begin{tabular}{cc}
\hline Score & Proportion of detail \\
\hline $\mathbf{0}$ & No enhancement visible \\
\hline $\mathbf{1}$ & Less than $1 / 3$ detail visible \\
\hline $\mathbf{2}$ & $1 / 3-2 / 3$ detail visible \\
\hline $\mathbf{3}$ & More than $2 / 3$ detail visible \\
\hline $\mathbf{4}$ & Fully detailed fingermark visible \\
\hline
\end{tabular}

\subsection{Statistical analysis}

All raw data was collected and entered into separate tables, each displayed ten scores (for each mark in a depletion) for each of the three donors, for a specific fingermark age and treatment used. The tables were analysed using the Microsoft Excel package and additional statistical analyses were carried out using custom scripts written in R [27]. 


\section{Results and Discussion}

The Home Office has published recommendations on the most effective sequential processes for mark enhancement on Bank of England polymer notes. However, no studies have been published on mark enhancement for CB and RBS polymer banknotes. The SPA Forensic Services can receive hundreds of thousands of pounds' worth of banknotes as part of a single case. The majority of these notes are Clydesdale banknotes, as they are the most common banknotes in circulation. This large number of banknotes received as evidence means that forensic scientists require an efficient and effective method of retrieving latent fingermarks from these notes. The exact make-up of polymer banknotes is proprietary, and each bank produces their notes differently; therefore, the same processing sequence may not be effective across all note types $[6,7,8,9,10]$. The main objective of this study was to determine the best processing techniques for fingermark enhancement on CB and RBS polymer banknotes.

Three key measures were statistically analysed: the score out of 4 for each visible mark, the total score out of 40 across a depletion series, and the total score out of 10 for each depletion series. Although some variability was evident between the two repeats carried out on consecutive days, the overall patterns seen were the same. Data from the two repeats were statistically compared (Table 3 ) and no significant differences were found between the two datasets; therefore, the results from the two repeats were combined.

Data on the scores out of four were considered across depletion series. Figure 10 shows the average score for each mark in the depletion series, for each time period, split by treatment and donor. 

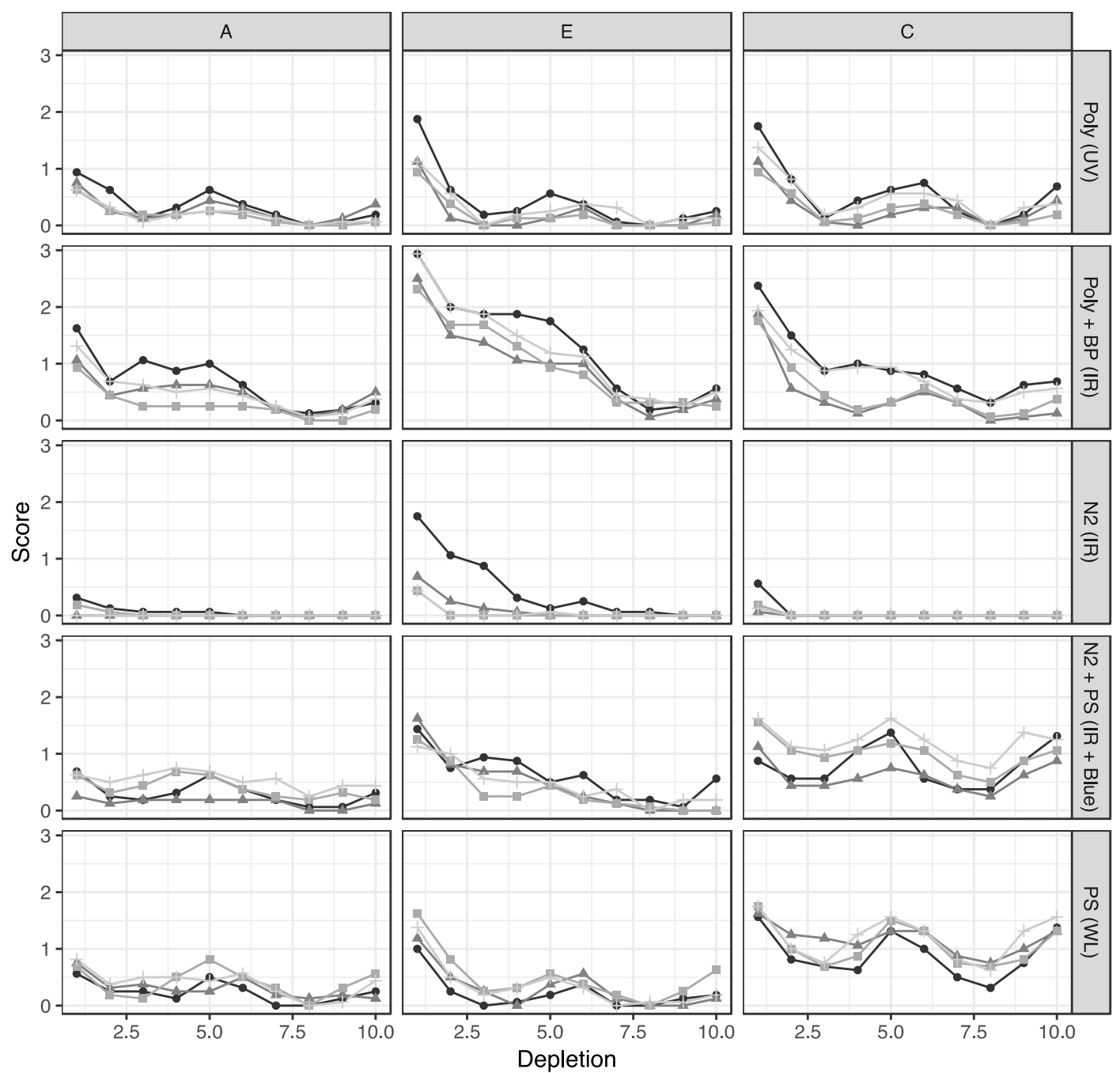

Figure 10. Mean score out of 4 across depletion series for marks enhanced using each technique (shown in panels down right-hand side of figure) and each donor (shown in panels across top of figure), split by time period.

In general, scores for fpNATURAL $® 2$ powder (N2) treatment were poorest, and decreased as the storage time period increased, but otherwise the time period had little effect on scores. The highest scores for PolyCyano UV (Poly) and PolyCyano UV followed by black magnetic powder (Poly+BP) were both at the start of the 10-mark depletion, and across the series showed a gradual decrease before levelling off. This is due to fingermarks deposited at the beginning of a depletion having a higher moisture content, therefore Poly and Poly+BP are more effective at enhancement. Powder suspension black (PS) also behaved as expected, exhibiting slightly increased scores for fingermarks aged 21 and 28 days. This is expected, as 
PS is more effective at enhancing older fingermarks [17]. PS + N2 showed a similar trend to PS alone, however the scores were lower on average. Although all treatments show a general decline over the depletion series, there is a small increase in score at the middle and end of many depletions. The beginning, middle and end of each depletion series are situated at the outer edges of each note. This increase is therefore due to the higher levels of background pattern interference in the middle of each note and is a useful observation, because individuals are more likely to handle the notes around the edges, and therefore more information is likely to be extracted from these marks. The transparent window of each note is located at one end of each note type, and the remaining surfaces of the notes are opaque. The higher scores observed at all the outer edges of the notes suggests that rather than being a result of fingermarks being more effectively recovered from transparent areas of the banknotes, the higher scores at the outer edges result from lower levels of background pattern interference in these areas.

In general, the results suggest there is a trend for scores to decrease across depletion series, and that this is more pronounced for better donors and better enhancement techniques. This means that marks within a depletion series are not independent of each other, and the position of an individual mark within a depletion series influence its score. In order to statistically analyse the data further, scores were therefore summed across each depletion series, to give a total score out of 40 , to account for the effect on score of position within a depletion series. In addition, summing scores across depletion series will also remove any impact of the positioning of individual marks within a depletion series on either transparent or opaque areas of the banknote.

A linear regression model was used to analyse the score out of 40 as a function of all other variables: enhancement treatment (N2, N2 + PS, Poly, Poly + BP, PS), donor (A, E, C), side (side 1 or 2 of the note), bank (CB or RBS), note type ( $£ 5$ or $£ 10)$, time period $(7,14,21,28$ days), and repeat (1 or 2). Significance was assessed using the anova function in $\mathrm{R}$, which computes analysis of variance tables. This is shown in Table 3 for these data. 
Table 3: Analysis of variance table for the linear model for score.

\begin{tabular}{cccccc}
\hline Variable & $\begin{array}{c}\text { Degrees of } \\
\text { freedom }\end{array}$ & $\begin{array}{c}\text { Sum of } \\
\text { squares }\end{array}$ & $\begin{array}{c}\text { Mean } \\
\text { square }\end{array}$ & F-value & P-value \\
\hline Treatment & 4 & 5721.24 & 1430.31 & 53.62 & $<0.001$ \\
\hline Donor & 2 & 1808.12 & 904.06 & 33.89 & $<0.001$ \\
\hline Note & 1 & 721.14 & 721.14 & 27.04 & $<0.001$ \\
\hline Bank & 1 & 265.44 & 265.44 & 9.95 & 0.002 \\
\hline Side & 1 & 215.10 & 215.10 & 8.06 & 0.005 \\
\hline Repeat & 1 & 2.92 & 2.92 & 0.11 & 0.741 \\
\hline Days & 1 & 0.46 & 0.46 & 0.02 & 0.896 \\
\hline
\end{tabular}

This analysis showed that there was no significant impact on the score out of 40 of either repeat or the fingermark age. However, all other variables had a significant impact on this score, most notably the donor, note type and enhancement treatment. The impact of each of these variables is considered in turn below.

\section{1 Donor variability, and storage time period}

The total scores per donor for each of the four time scales on all banknotes were collated, giving a score out of a possible overall total of 640 (i.e., a total of 160 per note type; 80 per note type multiplied by two for the repeat, multiplied by four for the different note types $=640$ ) (Figure 11). This addition of scores was done in order to remove the effect of variability between substrates. For all time periods, the scores produced using N2+PS were better than N2 alone. Similarly, the results using Poly improved with the addition of black magnetic powder (Poly+BP). There was no clear pattern in scores over time, with the majority of enhancement techniques producing relatively consistent scores over the four time periods, as expected from the statistical analysis reported above. The exception to this was the scores produced by N2 alone, which decreased over time. These results show that across all four time scales, most of the enhancement treatments give good results regardless of the age of the fingermark, between 7 and 28 days. 


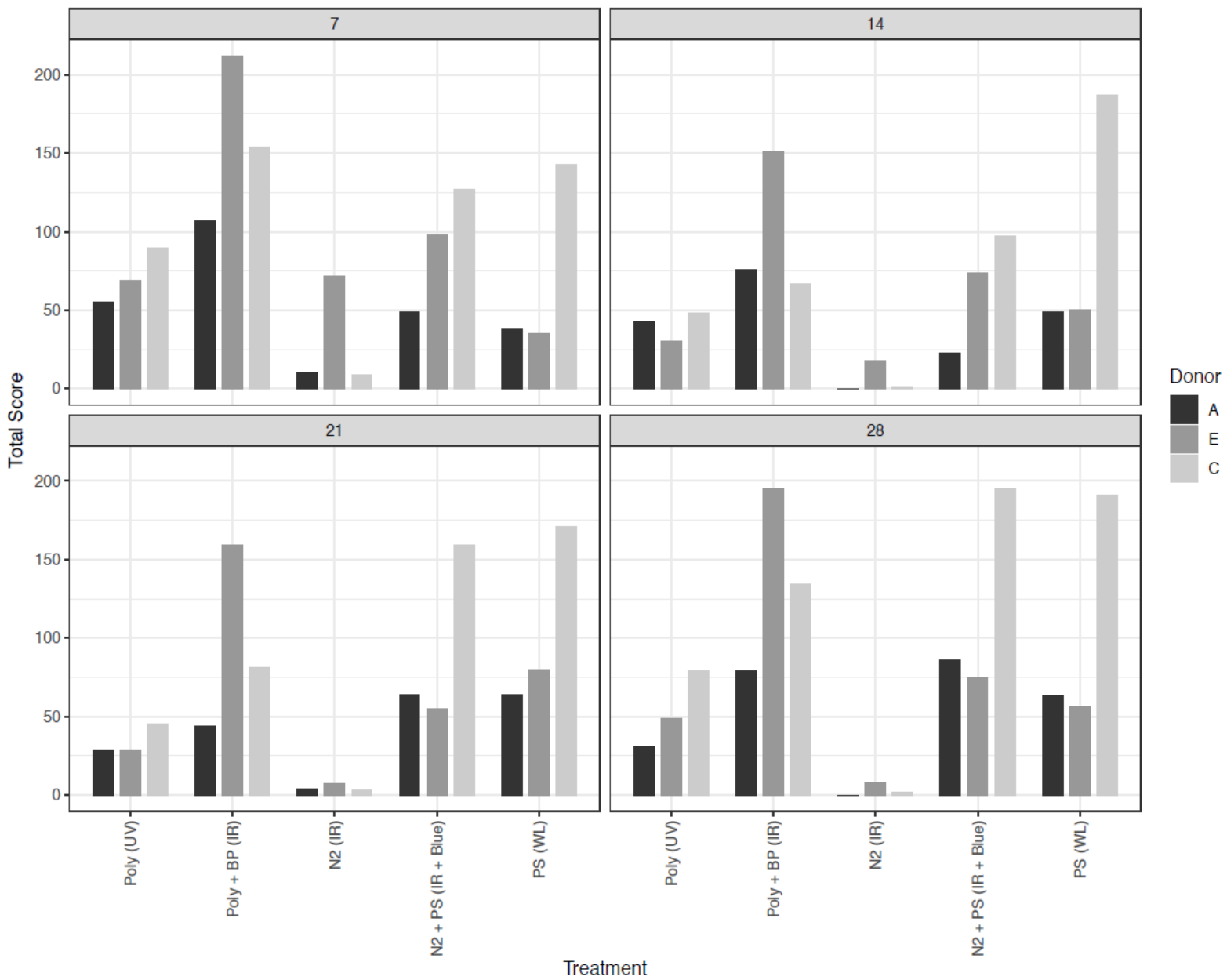

Figure 11. Total score (out of a maximum of 640) for each enhancement process for notes stored for 7, 14, 21 and 28 days before enhancement, split by donor $(A=$ poor, $E=$ medium, $C=$ good).

Donor A (poor donor) behaved as expected overall, giving the lowest scores across the majority of time periods and enhancement techniques. Donor C (good donor) showed variable results, but overall, they produced the highest scores for the majority of processes across all time periods. In contrast, Donor E (medium donor) did not behave as expected, as they produced higher results than Donor $\mathrm{A}$ and $\mathrm{C}$ for the Poly+BP and N2 techniques, across all four time periods. Donor $E$ is a good representative of an intermediate donor, suggesting that these techniques could be good choices for general use.

\subsection{Note type}

The impact of note type (£5/£10, CB/RBS) on fingermark score was considered next, using the total score out of 40 . This is summarised in Figure 12, showing scores for each donor, split by enhancement technique and note type. PS and Poly+BP gave the best results on all four banknote types, with N2+PS also generating good results on most note types. Poly and N2 
gave the poorest results across all note types, and also across all donors. When considering donor effects, PS gave the best results for Donor C (good) and Donor A (poor), whereas Poly+BP gave the best results for Donor E (medium). Overall, all processes were somewhat more effective on the $£ 10$ notes compared to the $£ 5$ notes.

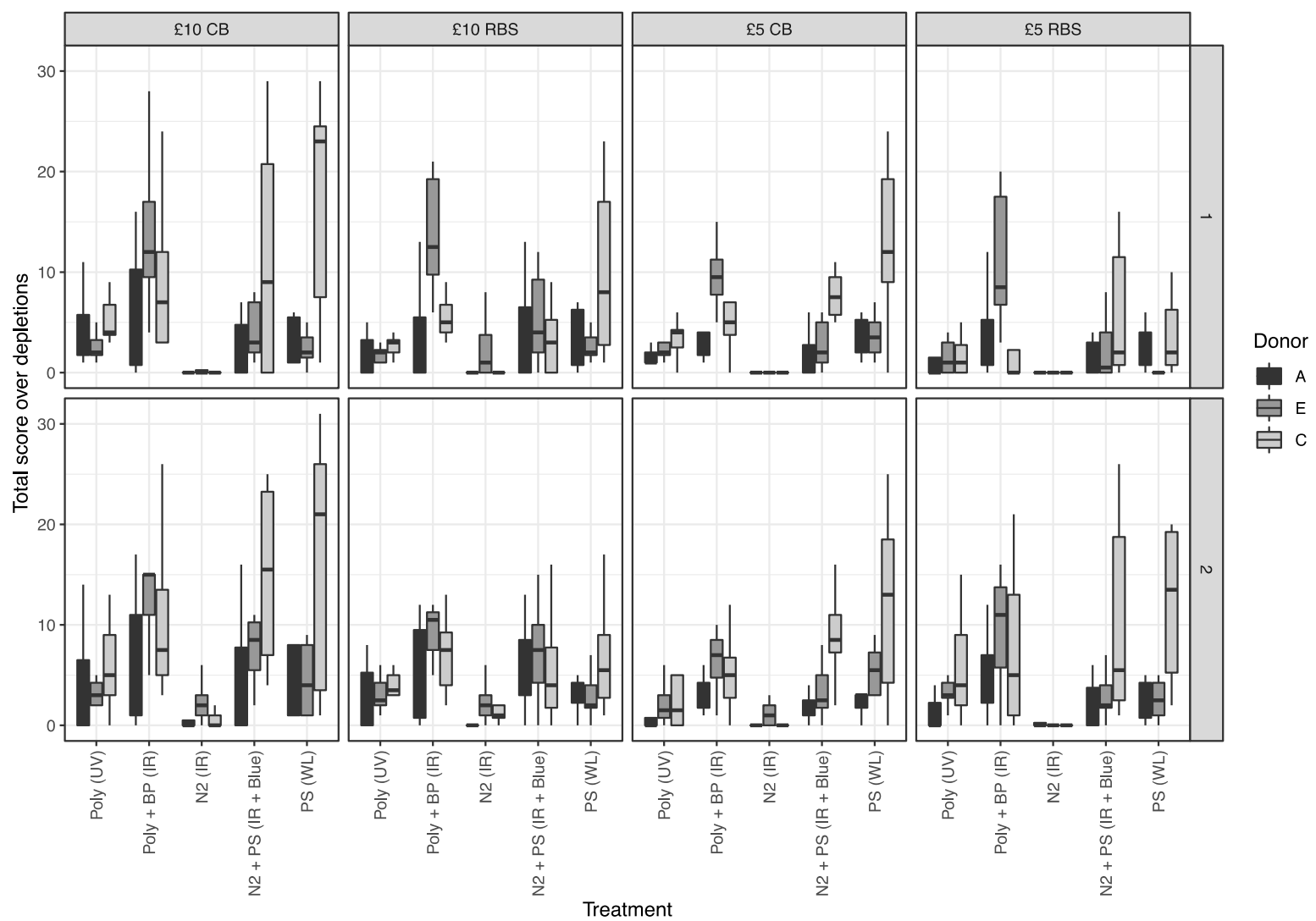

Figure 12. Comparison of the average depletion score for each of the four note types: $£ 10$ and $£ 5$ notes from Clydesdale Bank (CB) and Royal Bank of Scotland (RBS), for each enhancement technique and each donor. The top panels show side 1 of notes, the bottom panels show side 2.

\subsection{Comparison of Clydesdale Bank and Royal Bank of Scotland}

Fingermark scores were then compared to examine any difference in the results for each bank. Figure 13 shows the number of times each score (0-4) was assigned to a fingermark, for all $\mathrm{CB}$ and RBS notes across the whole study. The proportion of fingermarks given a score between 1 and 4 was somewhat higher on banknotes from CB (0.03) than on banknotes from RBS (0.27) (Fisher Exact Test P-value $=0.0066$, assuming independence of observations). This outcome is notable because the majority of banknotes received into the SPA Forensic Services laboratory are CB notes. Additionally, all enhancement processes except N2 powder 
were most effective on the CB banknotes compared to the RBS notes. This could also be due to differences in composition between banknote types, as each bank is likely to produce their notes slightly differently. For example, there could be differences in the inks used to print the banknotes from the two banks, and also in any surface treatments that may be required in order to make these inks bind to the surface of the notes [8].

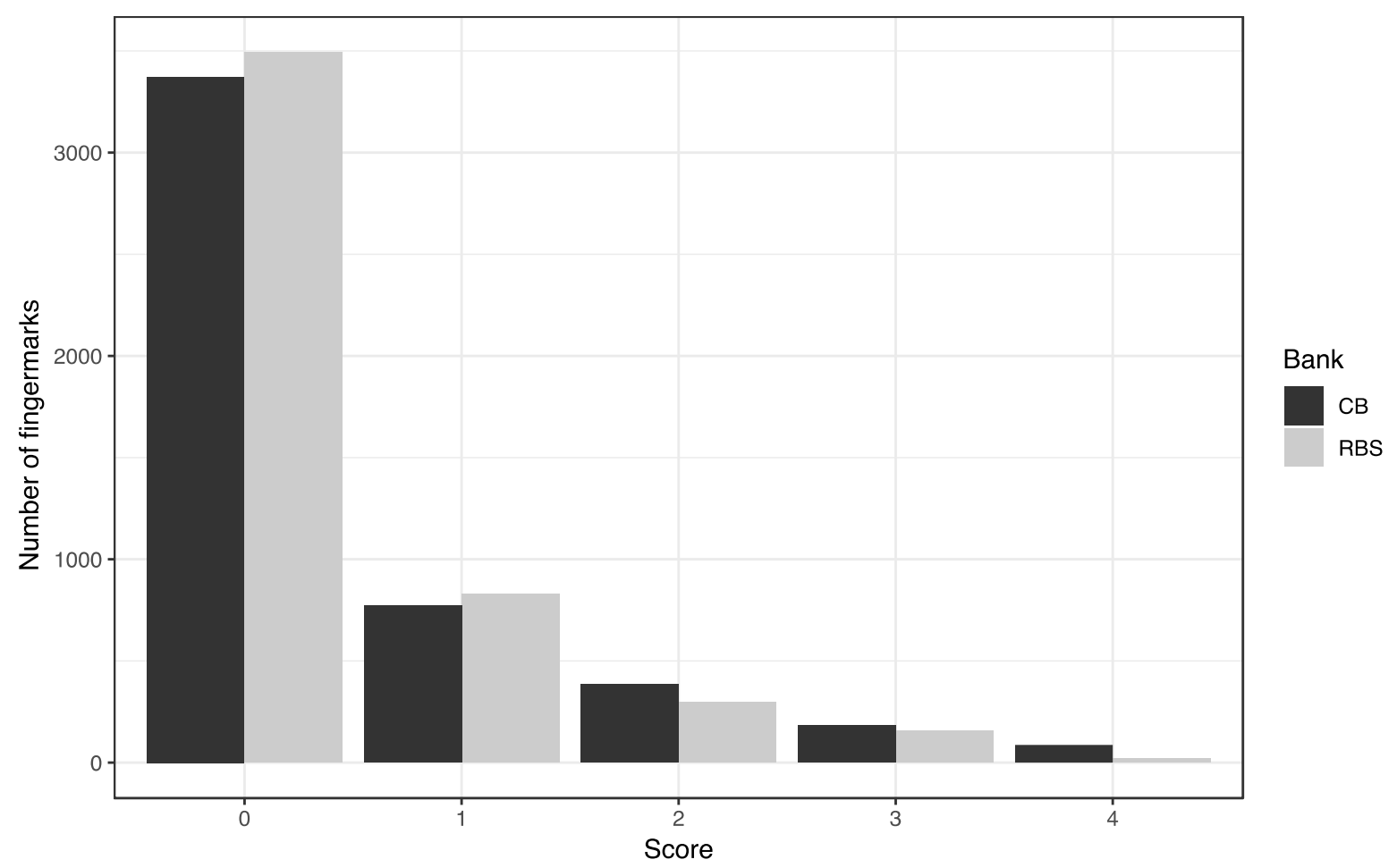

Figure 13. The number of marks assigned each of the five possible scores (0-4) for Clydesdale Bank (CB) and Royal Bank of Scotland (RBS) notes.

\subsection{Enhancement Techniques}

Fingermark scores were next considered for each of the single enhancement techniques (PS, N2, Poly), by summing scores across all note types, donors and repeat, giving a score out of a possible overall total of 1920 (Figure 14). Poly+BP was included here rather than Poly, as the black magnetic powder improved the visibility of marks compared to Poly alone. Although the black magnetic powder did not enhance any latent fingermarks that the superglue had not already adhered to, the powder in combination with the IR light helped with visualisation (Figure 15). All three processes behaved as expected on a non-porous surface; scores for marks enhanced using PS increased over time (PS works better on older marks), those for marks enhanced with $\mathrm{N} 2$ decreased over time, as fingermark powders are most effective on freshly deposited marks, and those for marks enhanced using Poly+BP varied over time. 


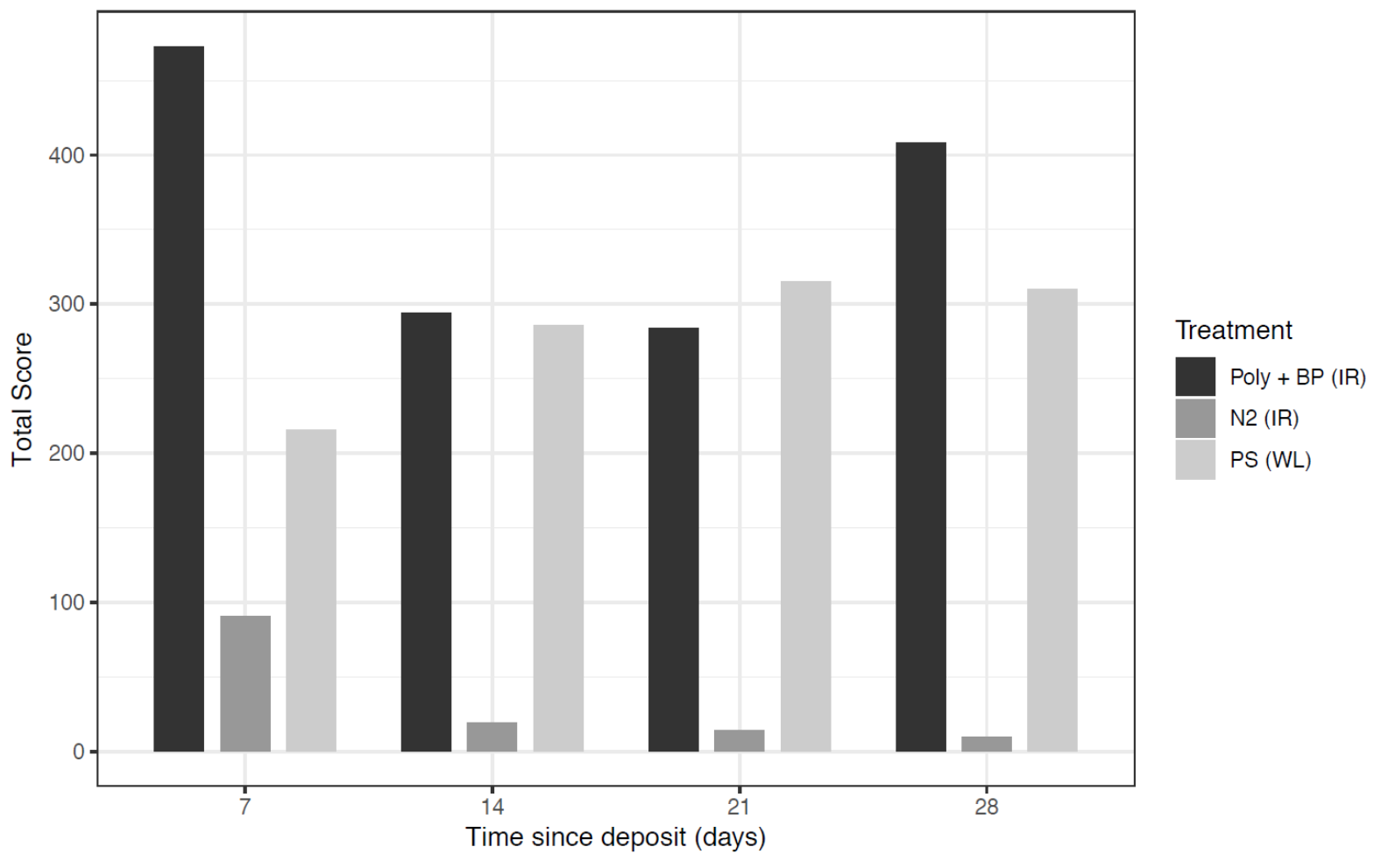

Figure 14. Total scores (out of a maximum of 1920) for each of the processes for each time period.
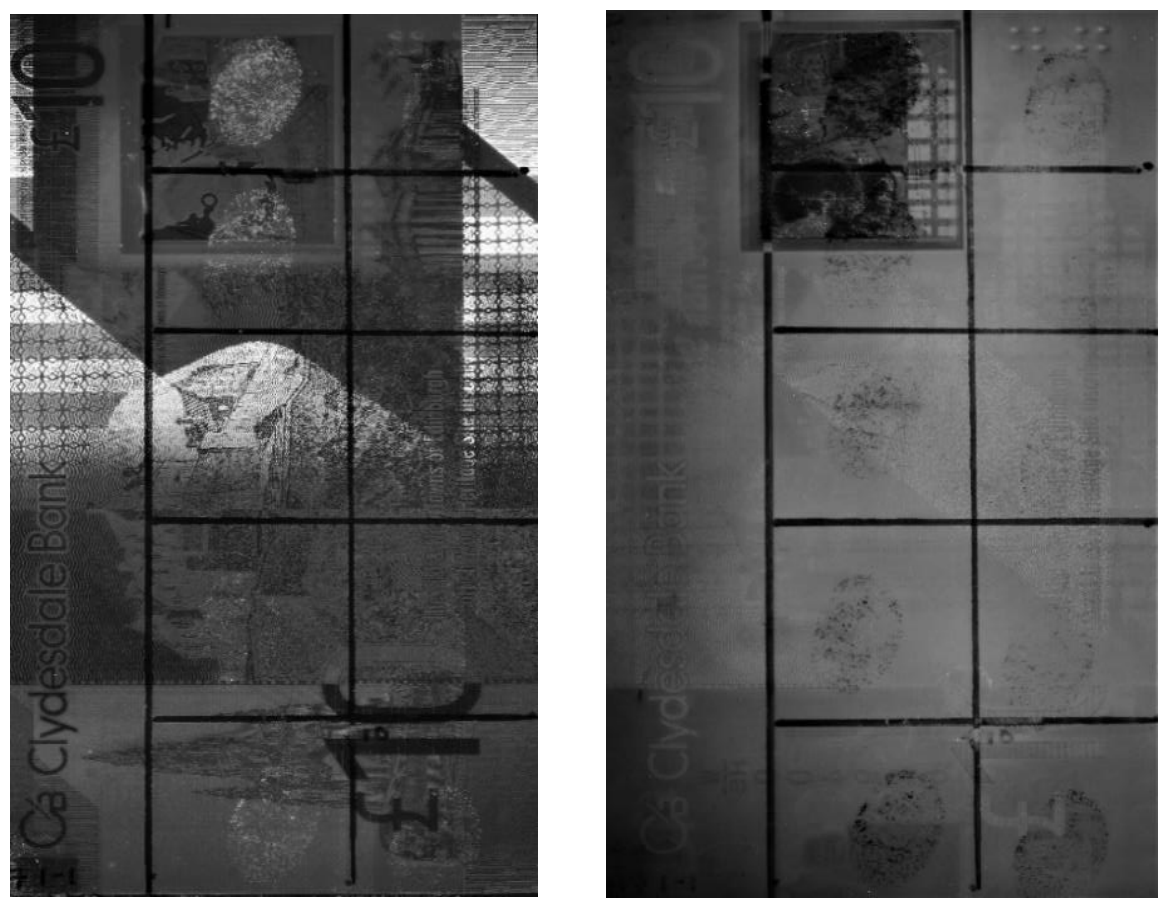

Figure 15. The same fingermarks enhanced using Poly + UV light $(350-380 \mathrm{~nm})$ (left) and Poly+BP + IR light $(730-800 \mathrm{~nm})$ (right). 
Across the experiment there was a highly significant impact of the enhancement process on fingermark scores. The complex backgrounds of all notes examined did present difficulties for all processes, making any fingermarks more difficult to visualise. However, the preliminary study carried out to determine the most effective light source for each processing sequence did help to eliminate the majority of background interference. Overall, Poly+BP was the most effective processing sequence followed by PS. In contrast, N2 produced the lowest results across all variables, followed by Poly. The addition of a second treatment to both of these techniques greatly improved the scores obtained, indicating that sequential processing can be an important strategy in the enhancement of fingermarks on polymer banknotes. However, PS on its own was the second most effective process overall, followed by N2+PS. A comparison between the scores for these processing sequences suggests that N2 powder inhibits PS, as all visible marks on notes enhanced with N2+PS had PS on the ridges rather than N2.

\subsection{Comparison of scoring methods}

Two types of scores were given across depletion series, as described in the Materials and Methods section. Firstly, the scores out of 4 for each mark were summed across a depletion series to give a total score out of 40 . A second score, out of 10 , was also given to each depletion series, indicating how many of the ten fingermarks in the depletion series gave a score of 1 or more, i.e., the number of samples that contained any evidence of a fingermark. The score given out of 40 is considered to be somewhat subjective to the examiner, whereas the score out of 10 is not. The second score removes any subjectivity from the results, so the two scores were compared in order to determine the impact of any subjectivity on the results.

The results displayed in Figure 16A show the mean scores out of 40 for each enhancement technique, split by banknote type and donor. Overall, the results show that Poly+BP gave the best results, followed by PS. The addition of a second treatment (i.e., black magnetic powder to Poly and powder suspension to fpNATURAL $® 2$ powder) improved the results compared to the initial treatment alone.

The results displayed in Figure 16B show the mean scores out of 10 for each enhancement technique, split by banknote type and donor. The results from this analysis mirror those results obtained from the scoring out of 40 , and confirm that Poly+BP gave the best results, followed by PS. This score should not be affected by any subjectivity on the part of the examiner since it reflects only whether fingermarks were visible or not, and so this comparison should indicate 
whether the score out of 40 is influenced by any subjectivity. The similarity between the two scores suggests that there is limited impact of subjectivity on fingermark scores. Both figures show the best enhancement technique for use on each banknote type; this analysis is important because in a casework scenario, the note type will be the only definitive factor known to the examiner. 
A

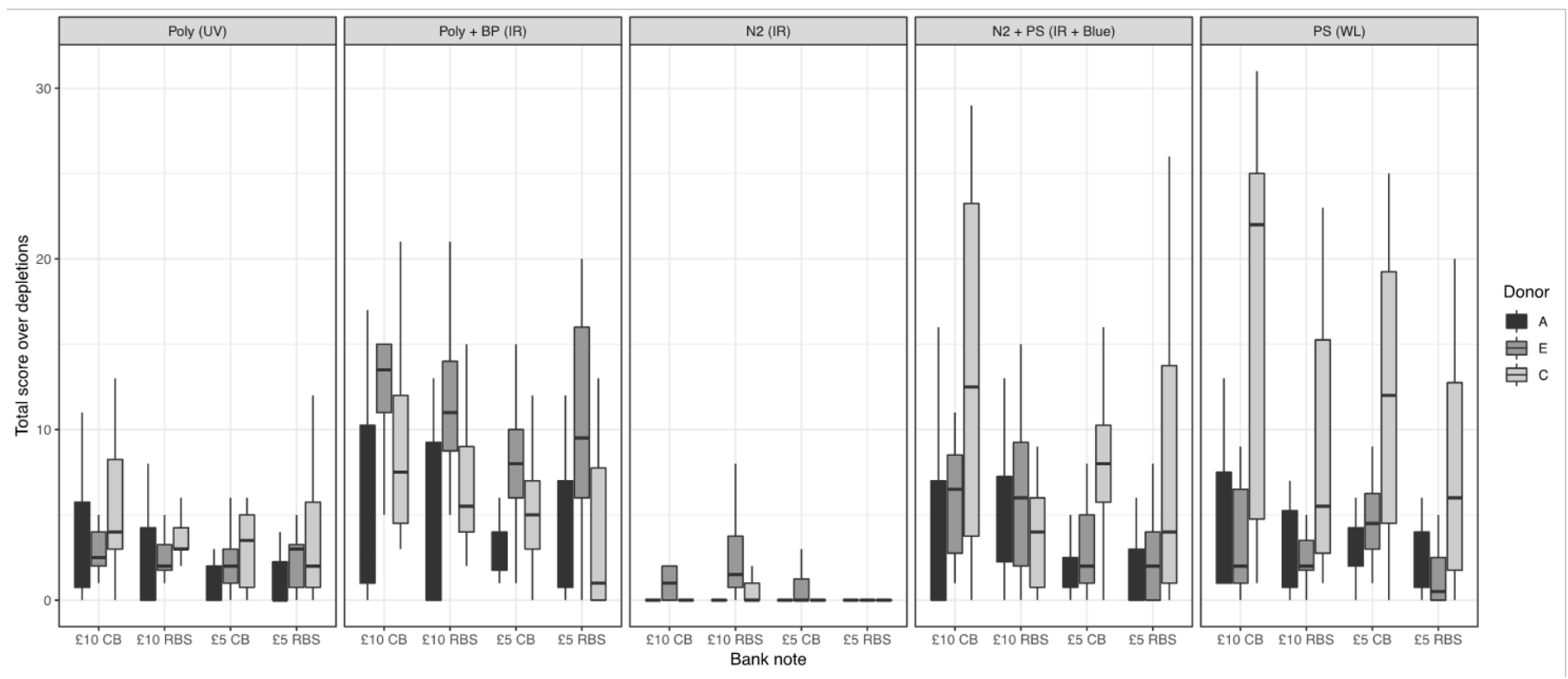

B

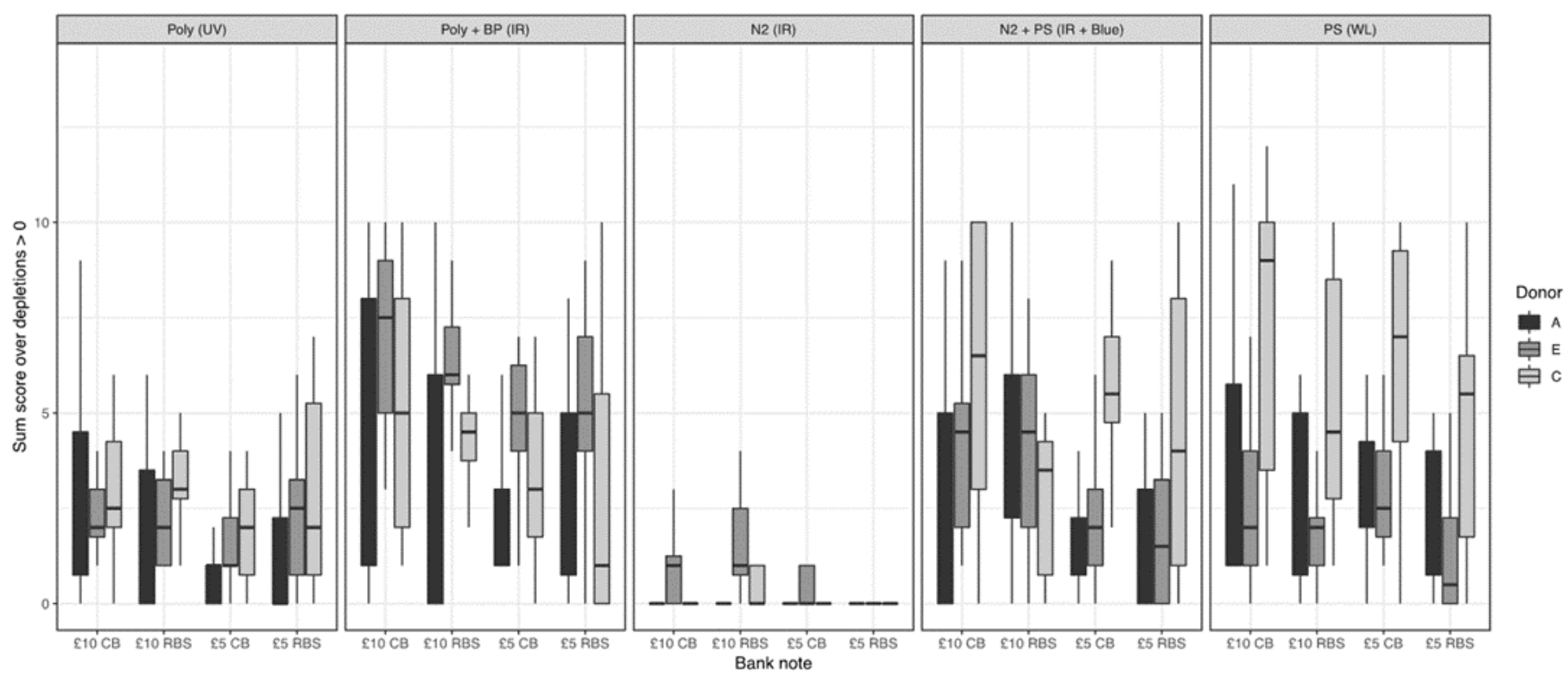

Figure 16. Mean total scores across depletions (A) out of 40 and (B) out of 10, for each enhancement technique, split by banknote type and donor. 


\subsection{Overall comparison of each enhancement technique}

For each enhancement technique, all scores out of 4 obtained for every fingermark were added together to carry out an overall comparison of the different techniques, with all other variables removed. Figure 17 shows this accumulated score, which combines the total score per donor, which would be a maximum of 640 , multiplied by three for all three donors, multiplied by four for all four time scales to give a total possible score of 7680 .

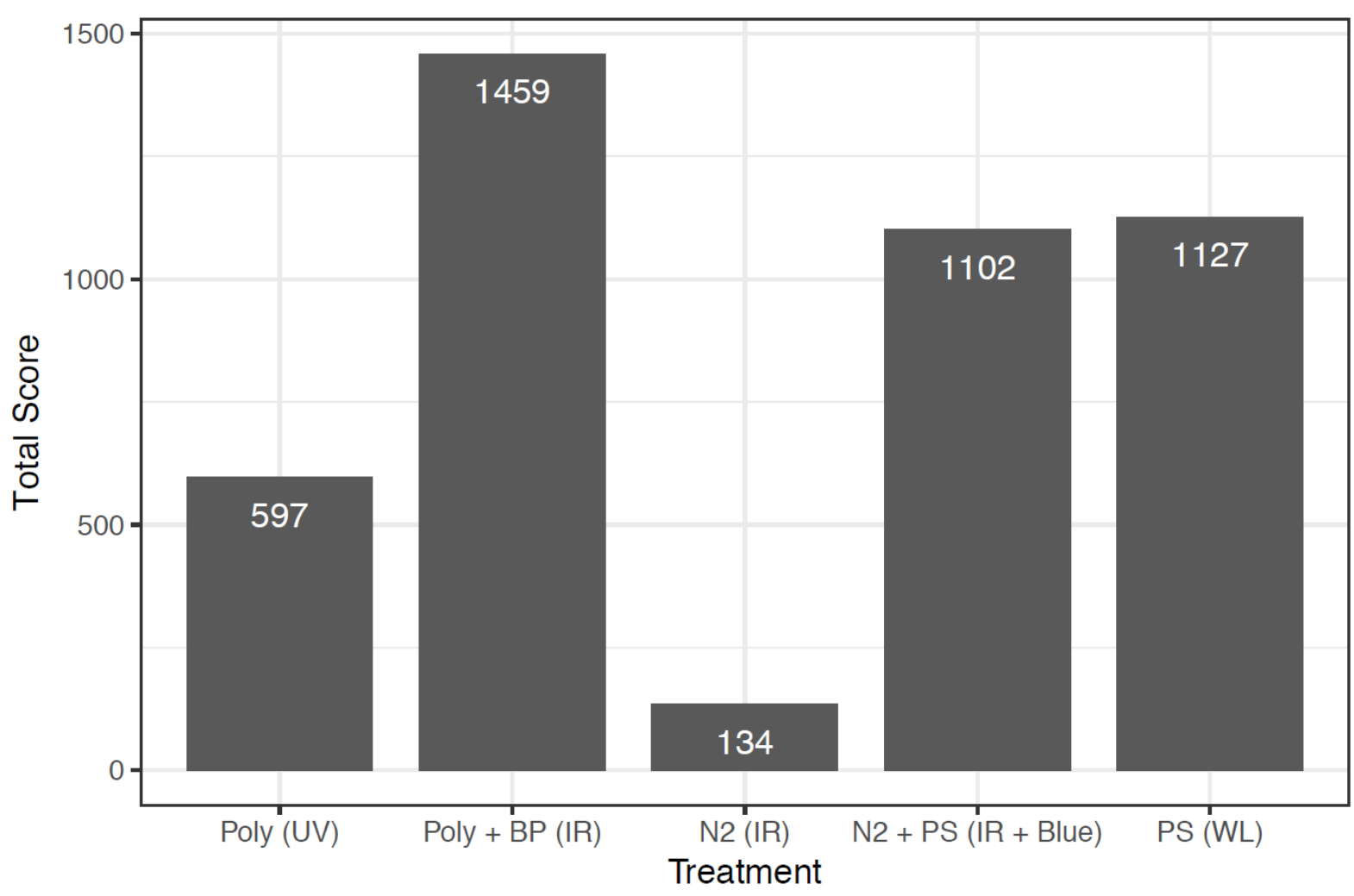

Figure 17. Total score (out of a maximum of 7680) across all marks for each enhancement technique.

Enhancement using Poly alone gave the second lowest score of 597. The addition of black magnetic powder (+BP) produced the highest accumulated score of 1459. This was followed by powder suspension (PS) with a score of 1127 , and fpNATURAL $® 2$ powder followed by powder suspension (N2+PS) with a score of 1102. fpNATURAL $® 2$ powder (N2) alone produced the lowest score (134). The raw data for N2 and N2+PS demonstrate that the use of N2 did not have any positive impact on scores. The addition of PS enhanced all of the marks that N2 had already enhanced; however, on top of this, it also enhanced marks that were not 
previously visible with $\mathrm{N} 2$ alone. All additional fingermarks that became visible with the addition of PS were due to the adherence of the PS and not the presence of N2.

\subsection{Further work}

This study was completed in a laboratory using three donors and uncirculated banknotes. Further work is recommended to test the effectiveness of these treatments on circulated banknotes in order to mimic a scenario more similar to that found in casework. A pseudooperational trial testing the two most effective enhancement techniques from this study is therefore now underway. The notes donated for use in this study were $£ 5$ and $£ 10$ Clydesdale Bank and Royal Bank of Scotland polymer notes. It is recommended that further work is carried out on both the $£ 20$ polymer notes once these have been released into circulation, and on Bank of Scotland polymer notes as these were not available for this study.

\section{Conclusions}

Five enhancement techniques or sequences were tested in order to determine which was the most effective for developing latent fingermarks on Clydesdale Bank and Royal Bank of Scotland $£ 5$ and $£ 10$ polymer banknotes. These were superglue fuming (using PolyCyano UV), with and without the addition of black magnetic powder, black iron oxide powder suspension, and infrared fluorescent powder (fpNATURAL ${ }^{2}$ ), with and without black powder suspension. There was limited impact of the age of fingermarks on the quality of enhanced marks, but there was a significant impact of note type, donor, and enhancement treatment.

All processes were most effective on the $£ 10$ notes from both banks, when compared to the $£ 5$ notes. Additionally, the majority of enhancement processes were most effective on $C B$ banknotes compared to RBS, and this is particularly important given that there are more $C B$ banknotes in circulation in Scotland than any other bank. The scores for marks from different donors (poor, medium, and good) were generally in line with expectations for the poor and good donors, but it was found that the medium donor produced the highest scores using superglue fuming and black magnetic powder enhancement treatment.

Currently, the recommendation for fingermark enhancement on English polymer banknotes is as follows: visual examination $>$ fluorescence examination $>$ powder $>$ IR reflection $>$ powder suspension $>\mathrm{IR}$ reflection $>$ gelatin lifting $[6,11,13]$. This differs from the FVM 
recommendation for polymeric currency: visual examination > fluorescence examination > superglue fuming $>$ vacuum metal deposition $>$ superglue fluorescent dye staining $>$ basic violet 3 , or black powder suspension $>$ basic violet 3 [17]. Since the release of Scottish polymer notes in 2016, the SPA Forensic Services has been using these guidelines for fingermark enhancement on Scottish polymer notes, with limited success. This study has determined that overall, enhancement of fingermarks was most successful using the following sequential process: visual examination > superglue fuming (using PolyCyanoUV) > black magnetic powder $>$ visual examination $>$ IR fluorescence examination, although black iron oxide powder suspension was also very effective when marks were not fresh. Superglue fuming and black magnetic powder is therefore recommended for enhancement of fingermarks on CB and RBS $£ 5$ and $£ 10$ polymer banknotes, and this process is now being implemented for use with these notes in the Scottish Police Authority Forensic Services Mark Enhancement Laboratory. 


\section{References}

[1] Hazarika, P. and Russell, D. Advances in Fingerprint Analysis. Angewandte Chemie International Edition, 51(15) (2012) 3524-3531.

[2] Bumbrah GS. Cyanoacrylate fuming method for detection of latent fingermarks: a review. Egyptian Journal of Forensic Sciences, 7 (2017) 4.

[3] Saferstein R. Criminalistics: An Introduction to Forensic Science. 11th ed. London: Pearson; (2015).

[4] Jackson ARW and Jackson JM. Forensic Science. 3rd ed. London: Pearson; (2011).

[5] McClintock R and Whymark R. Bank of England notes: the switch to polymer, Bank Engl. Quarterly Bulletin, 56(1) (2016) 23-32.

[6] Downham RP, Brewer ER, King RSP, Luscombe AM and Sears VG. Fingermark visualisation on uncirculated $£ 5$ (Bank of England) polymer notes: Initial process comparison studies. Forensic Science International. 275 (2017) 30-43.

[7] Jones N, Kelly M, Stoilovic M, Lennard C, Roux C. The Development of Latent Fingerprints on Polymer Banknotes. Journal of Forensic Identification. 53(1) (2003) 50-77.

[8] Guardian $1^{\mathrm{TM}}$ Facts and Figures, 15 September 2016. Available from: https://cclsecure.com/Guardian---Facts-and-Figures/, (accessed 28th February 2020).

[9] Downey, M. (2018) Email to Kenny Laing, 07 February 2018. Topic: Information on Polymer banknotes.

[10] Committee of Scottish Bankers. (2019). Polymer Banknotes. [online] Available at: https://www.scotbanks.org.uk/polymer-banknotes.html [Accessed 19th September 2018]. 
[11] PE INTERNATIONAL AG. The Bank of England - LCA of Paper and Polymer Bank Notes - Final Study Report. 2013. [Online] Available at:

https://www.bankofengland.co.uk/-/media/boe/files/banknotes/polymer/lca-of-paperand-polymer-bank-notes.pdf [Accessed $28^{\text {th }}$ February 2020].

[12] Lam, R., Wilkinson, D., Tse, T. and Pynn, B. Recommended Protocols for Fingerprint Detection on Canadian Polymer Banknotes - Part I: Chemical Development, Journal of Forensic Identification, 64(4) (2014) 375-401.

[13] Downham, R., Brewer, E., King, R. and Sears, V. Sequential processing strategies for fingermark visualisation on uncirculated $£ 10$ (Bank of England) polymer banknotes. Forensic Science International, 288 (2018) 140-158.

[14] Lennard, C. (2001). The Detection and Enhancement of Latent Fingerprints. [online] Latent-prints.com. Available at: http://latentprints.com/images/SpecialPresentation.pdf [Accessed 15 Jan. 2020].

[15] Dhall JK and Kapoor AK. Development of latent prints exposed to destructive crime scene conditions using wet powder suspensions. Egyptian Journal of Forensic Sciences 6 (2016) 396-404.

[16] Jones, BJ., Downham, R., and Sears, VG. Effect of substrate surface topography on forensic development of latent fingerprints with iron oxide powder suspension. Surface and Interface Analysis 42 (2010) 438-442.

[17] Bandey, HL, ed. Fingermark Visualisation Manual, $1^{\text {st }}$ ed. (2014), Home Office Centre for Applied Science and Technology: Sandridge.

[18] BVDA - Powder suspensions [Internet]. Bvda.com. 2018 [accessed $26^{\text {th }}$ February 2020]. Available from: http://www.bvda.com/en/powder-suspensions

[19] Champod, C., Lennard, C., Margot, P. and Stoilovic, M. Fingerprints and Other Ridge Skin Impressions, 2nd ed. (2016), CRC Press: Boca Raton.

[20] Home Office Centre for Applied Science and Technology. Fingermark Visualisation on Polymer Currency (Bank of England) (2017). Available from: 
https://www.gov.uk/government/publications/fingermark-visualisation-on-polymercurrency-bank-of-england

[21] Lee, H., Gaensslen, RE. Advances in Fingerprint Technology, 1st ed. (1991), Hoboken: CRC Press.

[22] Boyd, K. Comparison of cyanoacrylate enhanced with Basic Yellow 40 to the onestep fluorescent PolyCyano UV cyanoacrylate powder on various substrates. (2013). Unpublished (MSc) thesis, University of Strathclyde, Glasgow.

[23] Laing, K. FS-PHY-VAL-0510 (2013) Validation For The Use Of Fluorescent Superglue.

[24] International Fingerprint Research Group. Guidelines for the Assessment of Fingermark Detection Techniques. Journal of Forensic Identification, 64(2) (2014) 174-200.

[25] Sears, V., Bleay, S., Bandey, H., Bowman, V. A methodology for finger mark research. Science \& Justice. 52(3) (2012) 145-160

[26] Fosterfreeman.com. (2020). MVC/D PolyCyano. [online] Available at: http://www.fosterfreeman.com/fingerprint-evidence/296-mvc-fuming-cabinet-4.html [Accessed 29 Feb. 2020].

[27] R Core Team (2017) R: A language and environment for statistical computing. R Foundation for Statistical Computing, Vienna, Austria (https://www.R-project.org/). 